\title{
Field Assessment of Influence of Organic Fertilizers on Microbial Profile and Sustainable Maize Production in a Flood Plain in Nigeria
}

\author{
Christiana Utibe Etuk*, Matthew Albert Ekpo \\ Department of Microbiology, Faculty of Science, University of Uyo, Uyo, Akwa Ibom State, Nigeria \\ Email address: \\ etukchristiana2422@yahoo.com (C. U. Etuk), emacopron@yahoo.com (M. A. Ekpo) \\ ${ }^{*}$ Corresponding author
}

\section{To cite this article:}

Christiana Utibe Etuk, Matthew Albert Ekpo. Field Assessment of Influence of Organic Fertilizers on Microbial Profile and Sustainable Maize Production in a Flood Plain in Nigeria. Frontiers in Environmental Microbiology. Vol. 6, No. 3, 2020, pp. 27-34.

doi: $10.11648 /$ j.fem.20200603.11

Received: May 15, 2020; Accepted: June 2, 2020; Published: July 17, 2020

\begin{abstract}
The stabilization and utilization of organic fertilizers in flood plain for sustainable agriculture in the tropics was studied in field experiment conducted at Etinan wetland soil (EW) of Akwa Ibom State, Nigeria in the tropics during two cropping seasons (C. S.) to study the effects of two composted and stabilized organic fertilizers (poultry droppings, PD and cow dung, $\mathrm{CD}$ ) on the microbial density and structure, soil properties, growth and yield of corn (Zea mays) according to standard procedures. The two treatments plus control, (the unfertilized plots) were arranged in a randomized block design with two replications giving three main plots; poultry manured plots (PM), cow manured plots (CM) and the control plots, $\mathrm{C}$, which were sectioned into nine subplots on which was a total of 81 mounds on the study site. Results showed that PD and CD (450 $\mathrm{gm}^{2}=4500 \mathrm{kgha}^{-1}$ ) incorporated into the EW produced higher mean microbial density (Total heterotrophic bacterial counts, $\mathrm{THBC}=\log 7.636$ and 8.64 , total actinomycetes counts, $\mathrm{TAC}=\log 6.57 \& 6.62$, diazotrophic bacterial counts, $\mathrm{DBC}=\log 5.35 \&$ 5.50 and total fungal counts, TFC $=\log 5.38 \& 5.45 \mathrm{cfug}^{-1}$ ) in both fertilized plots during the 1 st \& 2 nd C. S respectively than in the control with $6.62 \& 7.49,5.59 \& 5.52,5.44 \& 5.54,4.5 \& 5.49 \mathrm{cfug}^{-1}$ of THBC, TAC, DBC and TFC respectively. It was also shown that $\mathrm{PD}$ and $\mathrm{CD}$ application into $\mathrm{EW}$ produced higher physicochemical properties, nutrient salts, compared to the $\mathrm{C}$. Growth/yield of the test crop, Zea mays were increased in the PM followed by CM compared to C in the EW during both C. S. Using the mean difference of two years, plants of PM had highest grain yield ( $4.16 \pm 0.16 \mathrm{t} t /$ acre $)$ compared to $(2.84 \pm 0.31 \mathrm{t} / \mathrm{acre})$ and $(0.09 \pm 0.23 \mathrm{t} / \mathrm{acre})$ of $\mathrm{CM}$ and $\mathrm{C}$ respectively. The effects of one time application of the organic fertilizers (without reapplication on the 2nd C. S) indicated higher crop harvest index, H. I. (0.63 and 0.64) of treatment plots compared to 0.19 and 0.20 of the $\mathrm{C}$. Therefore, utilization of PM to soils is recommended for sustainable crop production especially maize in the flood plain and in the tropics as a whole. It is also recommended that the $\mathrm{CD}$ could serve as a suitable substitute in the absence of PD. Wetland soils in the tropics should be converted from the hitherto wasteland to useful and sustainable arable lands with the utilization of stabilized and composted organic fertilizers.
\end{abstract}

Keywords: Organic Fertilizers, Maize Plant, Sustainable Production, Heterotrophic Bacteria, Flood Plain

\section{Introduction}

Agricultural activities have propelled the use and disposal of agrochemicals such as inorganic fertilizers into the environment with its attendant adverse effects on the environment (increased soil acidity, nutrient imbalance) as well as microbial activities, hence the need to adopt the less toxic fertilizer-the organic fertilizers e. g cow dung and poultry droppings (CD and PD). Devi, Sharma and Sighn [1] reported that utilization of animal manures (CD, PD etc.) as land fertilizer is an important disposal method as it contributes to diminishing environmental pollution from indiscriminate dumping of animal wastes. In Akwa Ibom State, Nigeria, the problem of appropriate disposal and reutilization of animal manures is being encountered Etuk [2]. Stabilization of organic wastes by composting provides an 
opportunity to reduce its bulk, odour, pathogens, toxicity and increasing the nutritive value Devi, Sharma and Sighn [1]. Sustainable crop production deals with keeping the soil alive with organic matter, integrated pest management and reduction in usage of chemical inputs, ensuring food safety and food quality, improving nutrient quality and fertilizing the soil with organic fertilizers Imadi, Shazadi, Gul and Hakeem [3]. Mixed microbial communities have biodegradative potential to degrade the organic compounds Mohapatra [4] present in manure. This research was done in a field experiment in a tropical flood plain located at Etinan, Akwa Ibom State, Nigeria during two cropping seasons to survey the stabilization and utilization of organic fertilizers in a wetland soil for sustainable agriculture in the tropics.

\section{Materials and Methods}

\subsection{Study Area}

The study area a tropical flood plain located in Etinan, designated EW (latitude $04^{\circ} 30$ and $5^{\circ} 30^{\prime} \mathrm{N}$ and longitude $07^{\circ} 30^{\prime}$ and $8^{\circ} 20^{\prime}$ East) of Akwa Ibom State, Nigeria (Figure 1). The climate is humid tropical, annual rainfall $(2500-3000 \mathrm{~mm})$, mean annual temperature (between 27 and $28^{\circ} \mathrm{C}$ ) and relative humidity (75-80\%) Imelda, Oshodeke and Akpan [5].

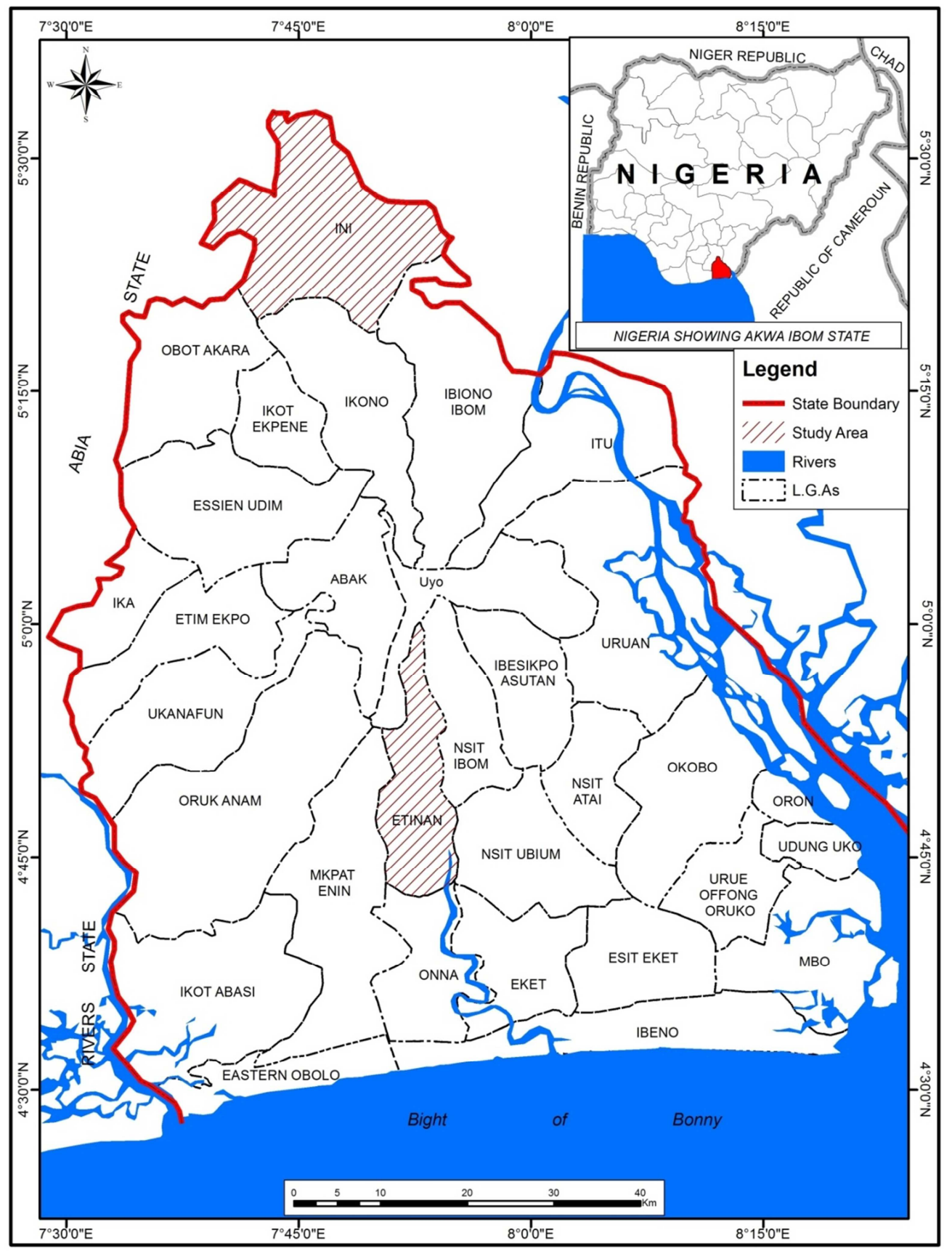

Source: Ministry of Lands and Town Planning, Akwa Ibom State.

Figure 1. The study site on the map of Etinan L. G. A., Akwa Ibom State, Nigeria. 


\subsection{Research Design}

The experiment was randomized complete block $\{3$ treatments (poultry manure and cow dung treatments and control) with 2 replications $\mathrm{x}$ six samplings $\}$ giving three plots which were subdivided into nine subplots on which were eighty-one mounds (each with a stand of maize plants) in the flood plain.

\subsection{Sampling Techniques}

\subsubsection{Collection of Organic Fertilizers}

Organic fertilizers CATTLE dung and poultry droppings were collected from a livestock market and private poultry farms in Uyo metropolis, Akwa Ibom State, Nigeria respectively.

\subsubsection{Collection of Test Crop and Soil Samples}

Maize (TZSR-W) seeds were collected from Akwa Ibom State Agricultural Development Programme (AKADEP) office. TZSR-W is Tropical Zea mays Streak Resistant White species. Homogenized soil samples were collected at depths of $0-10 \mathrm{~cm}$ and $20 \mathrm{~cm}$ biweekly for all analyses during first $\&$ second cropping seasons (1st \& 2nd C. S) Vinhal-Freitas, Wanger, Ferreira, Correa and Wendling [6].

\subsection{Analysis of Samples}

\subsubsection{Microbiological and Physicochemistry of Soil / Organic Fertilizers Samples}

Prior to cultivation the organic fertilizers were stabilized using the microbe-based active pile windrows composting methods of Mercola [7]. The soil and organic fertilizers were analyzed for their microbiological and physicochemical properties using standard methods of Dubey and Maheshwari, Robertson and Groffman [8, 11] and Traunfeld, [9] respectively at the beginning of the experiment Cenciani, Freitas, Critter and Airoldi [10] and bi-weekly Vinhal-Freitas, Wanger, Ferreira, Correa and Wendling [6] subsequently.

\subsubsection{Determination of Growth and Yield of Test Crop}

The growth parameters (leaf length, leaf-width, dry weight mass and height of plant aerial part) and yield of maize plants (number of grains per cob, weight of grains) were assessed according to methods of Agbogidi and Okonmah [12]. Maize was harvested fresh at 13 weeks after planting (WAP) Cenciani, Freitas, Critter and Airoldi [10] and the Harvest Index was evaluated after the methods of [19].

\subsection{Statistical Analysis}

The statistical package for Social Science version 20 (SPSS. 20) with level of significance maintained at $95 \%$ for each test was adopted for statistical analysis Sokal, and Rohlf [13].

\section{Results}

\subsection{Heterotrophic Microbial Populations in the Fresh / Composted Organic Fertilizers and in the Flood Plains During $1^{\text {st }}$ and $2^{\text {nd }}$ Cropping Seasons}

Results of the microbiological analyses of the fresh animal wastes (CD and PD), the composting animal wastes utilized $(\mathrm{CoCD}$ and $\mathrm{CoPD})$ and the soil samples revealed various counts of: (i) total heterotrophic bacteria (THBC), (ii) Actinomycetes (AC), (iii) diazotrophic bacteria (DBC), (iv) total fungi (TFC), (v) total coliform (TCC) and feacal coliform (FCC) at different age of the fertilizers throughout the twelve weeks of composting and cropping as shown in Table 1. It was revealed that composted poultry manure (CoPD) had highest THBC with $\log$ value of 7.41 followed by TAC $>$ DBC $>$ TFC and the least was TCC $\left(1.11 \mathrm{cfug}^{-1}\right)$. The mean counts difference between the microbial load in the CoPD and CoCD were mostly not statistically different at $\mathrm{P}=0.05$ except for the actinomycetes counts with mean difference between $\mathrm{CoCD}$ and CoPD being significant at $\mathrm{P}=0.05$.

During 1st C. S the results showed the following trend of microbial abundance (log transformed values) in the $\mathrm{EW}$ soils sampled: THBC (7.63) > AC (6.57), TFC (5.38) > DBC (5.35) $>$ TCC (Figures 2-5). Thus, from the results, the most abundant of the microbial groups were THBC while the least was TCC in EW. During 2nd C. S, the EW had similar pattern of microbial abundance as in the 1 st C. S was obtained (Figures 6-9).

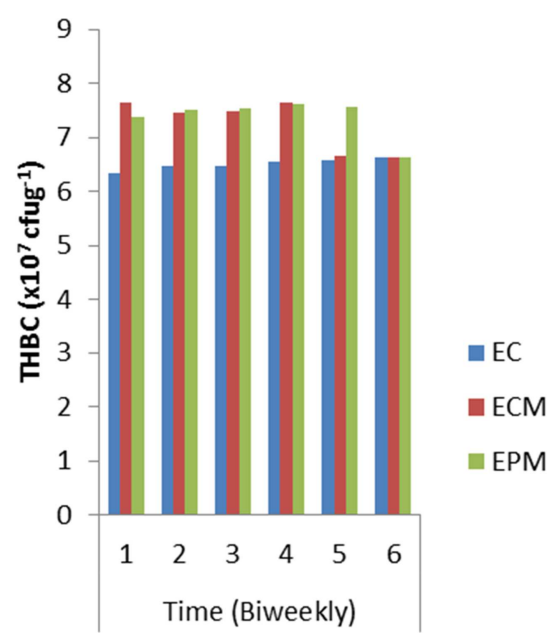

Figure 2. Total heterotrophic bacterial counts during $1^{\text {st }}$ C. S at Etinan flood plain.

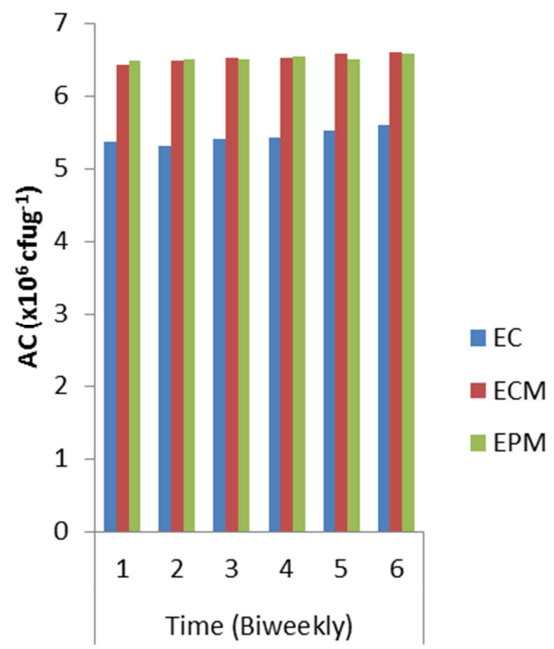

Figure 3. Actinomycetes counts during $1^{\text {st }}$ C. S at Etinan flood plain. 


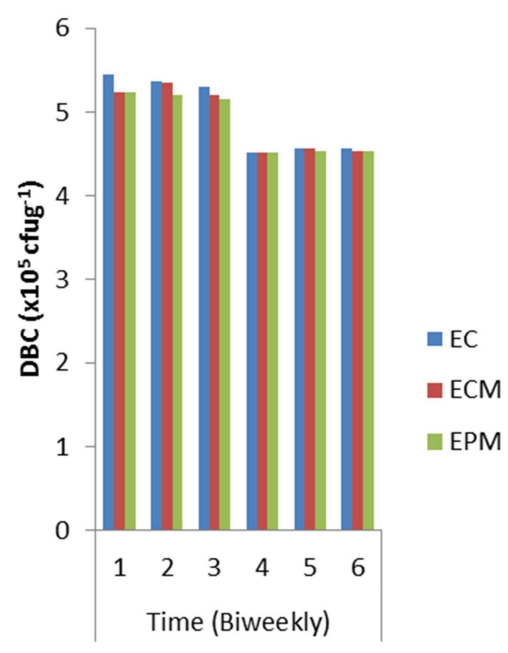

Figure 4. Diazotrophic bacterial counts during $1^{\text {st }}$ C. S at Etinan flood plain.

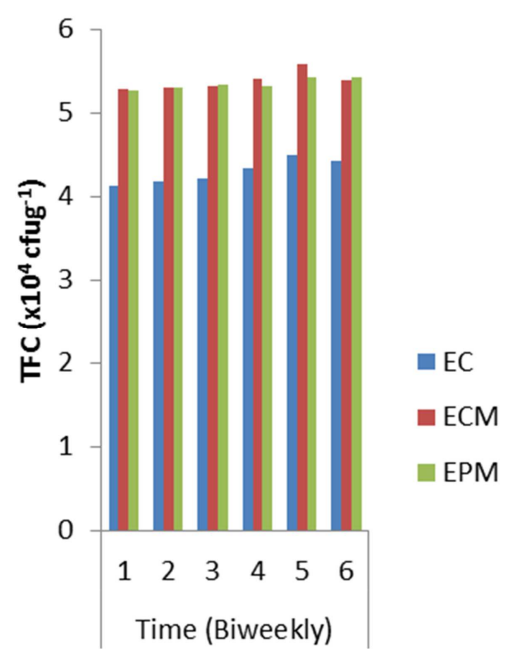

Figure 5. Total fungal counts during $1^{\text {st }}$ C. S at Etinan flood plain.

$\mathrm{THBC}=$ Total heterotrophic bacterial count, $\mathrm{AC}=$ Actinomycetes, $\mathrm{DBC}=$ diazotrophic bacterial, $\mathrm{TFC}=$ total fungal, $\mathrm{TCC}=$ total coliform counts, cfug $^{-1}=$ Colony forming unit per gram, EC, ECM, EPM and C. S=Etinan control, cow manured, poultry manured plots, cropping season, respectively.

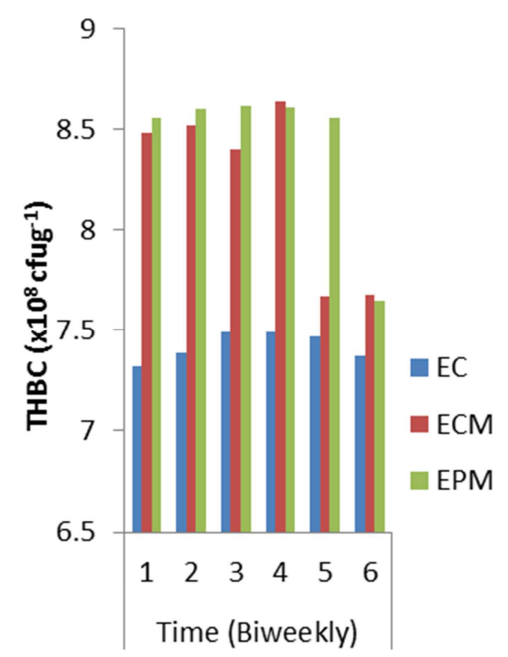

Figure 6. Total heterotrophic bacterial counts during $2^{\text {nd }}$ C. S at Etinan flood plain.

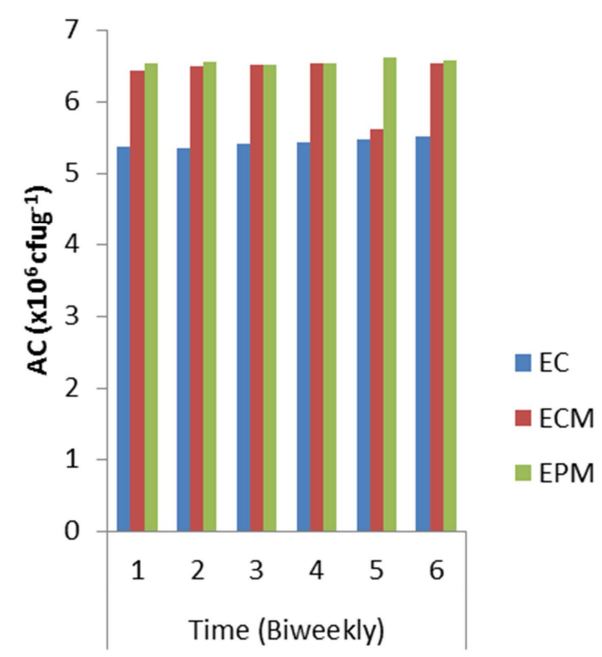

Figure 7. Actinomycetes counts during $2^{\text {nd }}$ C. S at Etinan flood plain.

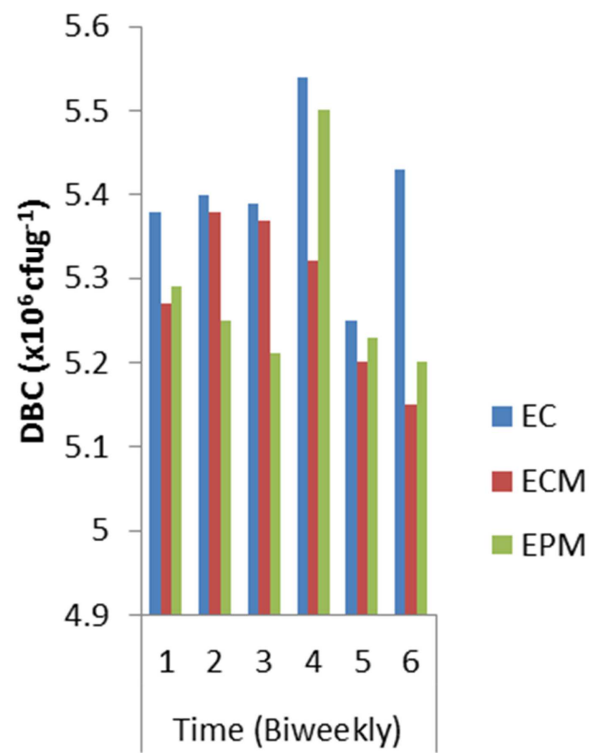

Figure 8. Diazotrophic bacterial counts during $2^{\text {nd }}$ C.S at Etinan flood plain.

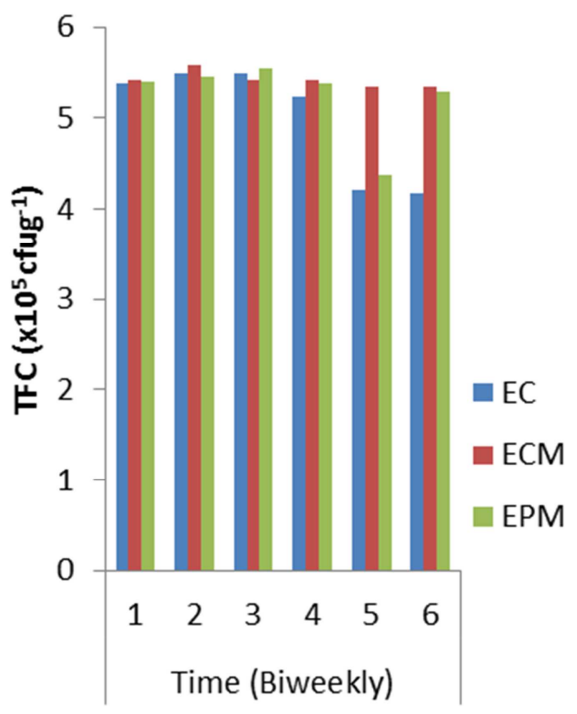

Figure 9. Total fungal counts during $2^{\text {nd }}$ C. S at Etinan flood plain. 
Bacterial spp. isolated include; Azotobacter chroococum, Alcaligens sp., Nocardia sp., Bacillus marcerans, Enterobacter aerogenes, Clostridium botulinum, Corynebacterium cc Enterococcus faecalis, Flavobacterium breve, Klebsiella pneumoniae, Micrococcus roseus, Nitrobacter sp., Nitrosomonas europea, Proteus mirabilis, Proteus vulgaris, Salmonella sp., Alcaligens sp, Pseudomonas aeruginosa strain B4 (Table 2).

Table 1. Microbial counts of organic fertilizers before and during composting

\begin{tabular}{|c|c|c|c|c|c|c|c|c|c|c|c|c|c|}
\hline $\begin{array}{l}\text { Sample } \\
\text { Code } \\
\end{array}$ & $\begin{array}{l}\text { Age } \\
\text { (Weeks) }\end{array}$ & $\begin{array}{l}\text { THBC }\left(\times 10^{7}\right. \\
\left.\text { cfug }^{-1}\right)\end{array}$ & $\begin{array}{l}\text { Log } \\
\text { Value } \\
\end{array}$ & $\begin{array}{l}\text { TFC (x10 } \\
\left.\text { cfug }^{-1}\right)\end{array}$ & $\begin{array}{l}\text { Log } \\
\text { Value } \\
\end{array}$ & $\begin{array}{l}\text { TCC }\left(\times 10^{3}\right. \\
\left.\text { cfug }^{-1}\right)\end{array}$ & $\begin{array}{l}\text { Log } \\
\text { Value } \\
\end{array}$ & $\begin{array}{l}\text { DBC }\left(\times 10^{5}\right. \\
\left.\text { cfug }^{-1}\right)\end{array}$ & $\begin{array}{l}\text { Log } \\
\text { Value } \\
\end{array}$ & $\begin{array}{l}\text { Total AC } \\
\left(\times 10^{6}\right) \mathrm{cfug}^{-1}\end{array}$ & $\begin{array}{l}\text { Log } \\
\text { Value } \\
\end{array}$ & $\operatorname{FCC}\left(\times 10^{2}\right)$ & $\begin{array}{l}\text { Log } \\
\text { Value }\end{array}$ \\
\hline FPD & 24 hours & $0.325 \pm 0.64$ & 6.51 & $0.169 \pm 0.65$ & 4.23 & $(0.53 \pm 0.35)$ & 1.72 & $(1.30 \pm 0.6)$ & 5.11 & $(1.69 \pm 0.05)$ & 5.23 & ND & \\
\hline $\mathrm{FCD}$ & & $0.231 \pm 0.30$ & 6.36 & $0.317 \pm 0.47$ & 4.50 & $(0.70 \pm 0.1)$ & 1.85 & $(1.34 \pm 0.17)$ & 5.13 & $(2.10 \pm 0.17)$ & 5.32 & $0.16 \pm 0.01$ & 1.20 \\
\hline \multirow[t]{6}{*}{$\mathrm{CoPD}$} & 2 & $0.261 \pm 0.44$ & 6.42 & $(0.210 \pm 0.10$ & 4.32 & $(0.13 \pm 0.06)$ & 1.11 & $(0.87 \pm 0.48)$ & 4.94 & $(1.13 \pm 0.42)$ & 5.05 & ND & \\
\hline & 4 & $2.570 \pm 1.0$ & 7.41 & $(2.74 \pm 1.49)$ & 5.44 & ND & & $(1.38 \pm 0.45)$ & 5.14 & $(1.90 \pm 1.31)$ & 5.28 & ND & \\
\hline & 6 & $2.020 \pm 0.08$ & 7.31 & $(2.43 \pm 1.03)$ & 5.39 & ND & & $(1.97 \pm 0.61)$ & 5.29 & $(3.87 \pm 1.76)$ & 5.59 & ND & \\
\hline & 8 & $0.187 \pm 1.01$ & 6.27 & $(2.57 \pm 0.8)$ & 4.41 & ND & & $(1.47 \pm 0.02)$ & 5.17 & $(2.70 \pm 0.40)$ & 5.43 & ND & \\
\hline & 10 & $0.203 \pm 0.06$ & 6.31 & $(2.83 \pm 1.13)$ & 4.45 & ND & & $(2.04 \pm 0.01)$ & 5.31 & $(3.29 \pm 0.71)$ & 5.52 & ND & \\
\hline & 12 & $0.202 \pm 0.92$ & 6.32 & $(2.18 \pm 0.24)$ & 5.34 & ND & & $(1.85 \pm 0.65)$ & 5.27 & $(2.43 \pm 0.06)$ & 5.39 & ND & \\
\hline \multirow[t]{5}{*}{$\mathrm{CoCD}$} & 2 & $0.247 \pm 0.2$ & 6.39 & $(2.90 \pm 1.67)$ & 4.67 & $(0.27 \pm 0.16)$ & 1.43 & $(0.73 \pm 0.55)$ & 4.86 & $(2.87 \pm 1.47)$ & 5.46 & $0.40 \pm 0.03$ & 1.60 \\
\hline & 4 & $2.330 \pm 0.35$ & 7.37 & $(3.23 \pm 1.03)$ & 5.55 & $(0.17 \pm 0.06)$ & 1.23 & $(0.91 \pm 0.33)$ & 4.96 & $(1.90 \pm 0.70)$ & 5.28 & ND & \\
\hline & 6 & $2.260 \pm 0.17$ & 7.35 & $(2.63 \pm 0.40)$ & 5.42 & ND & & $(0.21 \pm 0.19)$ & 4.32 & $(2.20 \pm 0.78)$ & 5.34 & ND & \\
\hline & 10 & $0.246 \pm 0.53$ & 6.39 & $(2.18 \pm 1.08)$ & 4.50 & ND & & $(0.117 \pm 0.09)$ & 4.07 & $(2.24 \pm 1.76)$ & 5.35 & ND & \\
\hline & 12 & $2.310 \pm 0.60$ & 7.36 & $(2.10 \pm 0.15)$ & 4.42 & ND & & $(0.102 \pm 1.22)$ & 4.01 & $(2.10 \pm 1.4)$ & 5.32 & ND & \\
\hline
\end{tabular}

$\mathrm{FPD}, \mathrm{FCD}=$ fresh poultry and cow manures respectively; 2 - 12=biweekly; $\mathrm{CoPD}, \mathrm{CoCD}=\mathrm{Composting}$ poultry and cow manures.

Table 2. Microbial isolates obtained from Etinan floodplain during 1 st and 2 nd cropping seasons, their density and their percentage prevalence.

\begin{tabular}{|c|c|c|c|c|c|c|c|c|c|c|c|c|c|}
\hline & \multirow{3}{*}{ Isolates (Bacterial) } & \multicolumn{4}{|l|}{$\mathbf{E C}$} & \multicolumn{4}{|c|}{ ECM } & \multicolumn{4}{|l|}{ EPM } \\
\hline & & \multicolumn{2}{|c|}{$\begin{array}{l}\text { No. of } \\
\text { Colonies }\end{array}$} & \multicolumn{2}{|c|}{ Prevalence (\%) } & \multicolumn{2}{|c|}{$\begin{array}{l}\text { No. of } \\
\text { Colonies }\end{array}$} & \multicolumn{2}{|c|}{ Prevalence (\%) } & \multicolumn{2}{|c|}{ No. of Colonies } & \multicolumn{2}{|c|}{ Prevalence (\%) } \\
\hline & & 1st & 2nd & 1st & 2nd & 1st & 2nd & 1st & 2nd & 1st & 2nd & 1st & 2nd \\
\hline 1 & Azotobacter chroococum. & 20 & 20 & 2.7 & 3.68 & 22 & 29 & 2.19 & 2.22 & 18 & 16 & 1.5 & 0.99 \\
\hline 3 & Pseudomona aeruginosa. & 110 & 59 & 14.85 & 10.85 & 150 & 164 & 14.93 & 12.41 & 141 & 169 & 11.75 & 10.48 \\
\hline 4 & Flavobacterium breve. & 96 & 60 & 12.96 & 11.03 & 131 & 102 & 13.03 & $7 . .72$ & 152 & 70 & 12.67 & 7.44 \\
\hline 5 & Alcaligenes sp. & 30 & 21 & 4.05 & 3.86 & 33 & 130 & 3.28 & 9.81 & 116 & 140 & 12.17 & 9.92 \\
\hline 6 & Micrococcus sp. & 30 & 41 & 4.05 & 7.54 & 127 & 149 & 12.64 & 11.28 & 84 & 102 & 7.00 & 6.32 \\
\hline 7 & Corynebacterium bovis, & 44 & 43 & 5.94 & 7.9 & 27 & 122 & 2.69 & 9.24 & 47 & 69 & 3.92 & 4.28 \\
\hline 8 & Bacillus marcerans & 106 & 80 & 14.3 & 14.71 & 101 & 138 & 10.05 & 10.45 & 127 & 140 & 10.58 & 8.68 \\
\hline 9 & Enterococcus sp. & 48 & 21 & 6.48 & 3.86 & 33 & 75 & 3.28 & 5.68 & 58 & 50 & 4.83 & 9.3 \\
\hline 10 & Klebsiella sp. & 40 & 23 & 5.4 & 4.23 & 25 & 37 & 2.49 & 2.89 & 58 & 43 & 4.83 & 2.67 \\
\hline 11 & Alcaligens eutrophus & 106 & 72 & 14.3 & 13.24 & 123 & 92 & 12.24 & 6.96 & 185 & 190 & 15.42 & 11.78 \\
\hline 12 & Nitrobacter sp. & 18 & 18 & 2.43 & 3.3 & 10 & 9 & 0.99 & 0.68 & 10 & 12 & 0.83 & 0.74 \\
\hline 16 & Proteus mirabilis & 10 & 12 & 1.35 & 2.21 & 70 & 85 & 6.97 & 6.43 & 29 & 108 & 2.42 & 6.7 \\
\hline 17 & Proteus vulgaris & 10 & 10 & 1.35 & 1.84 & 55 & 73 & 5.47 & 5.53 & 18 & 50 & 1.5 & 9.3 \\
\hline 18 & Salmonella sp & 27 & 14 & 3.64 & 2.57 & 35 & 63 & 3.48 & 4.77 & 92 & 96 & 7.67 & 5.95 \\
\hline 19 & Nocardia sp. & 10 & 12 & 1.35 & 2.21 & 25 & 19 & 2.49 & 1.44 & 30 & 50 & 2.5 & 3.09 \\
\hline \multirow[t]{2}{*}{20} & E. aerogenes & 13 & 20 & 1.75 & 3.68 & 25 & 20 & 2.49 & 1.51 & 20 & 20 & 1.67 & 1.24 \\
\hline & Total & 741 & 546 & $100 \%$ & 100 & 1005 & 1321 & 100 & 100 & 1200 & 1613 & $100 \%$ & 100 \\
\hline 1 & Isolates (Fungal) & 6 & 10 & 6 & 8.6 & 8 & 14 & 6.6 & 6.63 & 12 & 24 & 7.45 & 10.76 \\
\hline 2 & Penicillium sp. & 12 & 14 & 12 & 12.1 & 18 & 28 & 13.9 & 13.27 & 10 & 16 & 6.21 & 7.17 \\
\hline 3 & Aspergillus fumigatus & 9 & 11 & 9 & 9.5 & 10 & 22 & 8.2 & 10.42 & 13 & 23 & 8.07 & 10.31 \\
\hline 4 & Fusarium sp. & 14 & 29 & 14 & 25 & 6 & 2 & 4.9 & 0.94 & 8 & 1 & 4.97 & 0.45 \\
\hline 5 & Mucorsp. & 6 & 9 & 6 & 7.8 & 2 & 27 & 1.6 & 12.79 & 17 & 18 & 10.56 & 8.07 \\
\hline 6 & Aspergillus flavus & 7 & 10 & 7 & 8.6 & 11 & 18 & 9 & 8.53 & 18 & 27 & 11.18 & 12.11 \\
\hline 7 & Rhizopus sp & 2 & 4 & 2 & 3.4 & 11 & 12 & 9 & 5.68 & 13 & 15 & 8.07 & 6.73 \\
\hline 8 & Saccharomyces sp. & 13 & 8 & 13 & 6.9 & 10 & 16 & 8.2 & 7.58 & 14 & $20 \mathrm{~s}$ & 8.7 & 8.97 \\
\hline 9 & Trichoderma sp. & 16 & 8 & 16 & 6.9 & 27 & 41 & 22.1 & 19.43 & 28 & 36 & 17.39 & 16.14 \\
\hline \multirow[t]{2}{*}{12} & Alternaria alternaria & 1 & 2 & 1 & 1.7 & 1 & 1 & 0.8 & 0.47 & 1 & 1 & 0.62 & 0.45 \\
\hline & Total & 100 & 116 & 100 & 100 & 122 & 211 & 100 & 100 & 161 & 223 & 100 & 100 \\
\hline
\end{tabular}




\subsection{The Occurrence of Microbial Isolates in Fresh, Composted Organic Fertilizers and Flood Plain Samples During $1^{\text {st }}$ and $2^{\text {nd }}$ Cropping Seasons}

In the EW soil samples the bacteria with highest prevalence (15.42 and 14.71\%) during 1st and 2nd C. S respectively were Alcaligenes eutrophus and Bacillus sp. from EPM and EC respectively while the bacteria with least percentage of occurrence $(0.83 \& 0.68 \%)$ was Nitrobacter $\mathrm{sp}$. isolated from both EPM and ECM during 1st and 2nd C. S respectively (Table 2). Fungal isolates with highest percentage prevalence (22.1 \& 19.43\%) was Trichoderma sp. from ECM soil samples, during 1 st $\& 2$ nd C. S respectively (Table 2).

\subsection{The Physicochemical Properties of the Flood Plain}

The mean values of the physicochemical properties of the EW (Table 3) during 1st C. S revealed as follows: highest mean temperature $\left(30.81 \pm 0.11^{\circ} \mathrm{C}\right)$ in $\mathrm{EPM}$ plot, $\mathrm{pH}$ value of the initial (EBSO) and EC soils were acidic (5.55 \pm 0.04 and $5.21 \pm 0.05$ ) but the $\mathrm{pH}$ of the treated plots during both seasons increased to neutral $(7.48 \pm 0.07)$ at ECM. Electrical conductivity was lower in the treated soil. Higher values of total organic carbon, organic matter, base saturation, total nitrogen, and nutritive salts were obtained in treatment plots than in the EC plots. However, there was slight decrease in concentrations of available phosphorous in the ECM plots than in the EC during 2nd C. S (Table 3).

Table 3. Physicochemical properties measured at Etinan floodplain in the 0-15 cm surface layer and core sample during 1st and 2nd cropping seasons.

\begin{tabular}{|c|c|c|c|c|c|c|c|}
\hline \multirow{2}{*}{ Properties } & \multicolumn{7}{|l|}{ site } \\
\hline & EBSO $_{1}$ & EC1 & EC2 & EPM1 & ЕРМ2 & ECM1 & ECM2 \\
\hline Temp $\left({ }^{\circ} \mathrm{C}\right)$ & $29.33 \pm 0.01$ & $29.07 \pm 0.04$ & $29.94 \pm 0.12$ & $30.81 \pm 0.11$ & $30.22 \pm 0.02$ & $30.00 \pm 0.18$ & $29.90 \pm 0.18$ \\
\hline $\mathrm{pH}\left(\mathrm{H}_{2} \mathrm{O}\right)$ & $5.55 \pm 0.04$ & $5.21 \pm 0.05$ & $5.83 \pm 0.07$ & $6.56 \pm 0.07$ & $6.77 \pm 0.06$ & $6.06 \pm 0.04$ & $7.48 \pm 0.07$ \\
\hline $\mathrm{EC}\left(\mathrm{mScm}^{-1}\right)$ & $3.29 \pm 0.03$ & $3.52 \pm 0.08$ & $3.51 \pm 0.02$ & $2.87 \pm 0.02$ & $3.18 \pm 0.03$ & $2.72 \pm 0.02$ & $3.05 \pm 0.02$ \\
\hline $\mathrm{BS}(\%)$ & $50.40 \pm 0.13$ & $55.32 \pm 0.22$ & $57.17 \pm 0.12$ & $57.17 \pm 0.42$ & $61.61 \pm 0.22$ & $58.02 \pm 0.12$ & $63.98 \pm 0.19$ \\
\hline Moisture content $(\%)$ & $42.90 \pm 0.20$ & $42.47 \pm 0.09$ & $42.84 \pm 0.10$ & $53.97 \pm 0.12$ & $54.86 \pm 0.11$ & $53.53 \pm 0.09$ & $55.01 \pm 0.14$ \\
\hline TOC $(\%)$ & $1.21 \pm 0.01$ & $1.10 \pm 0.01$ & $0.76 \pm 0.03$ & $1.59 \pm 0.02$ & $2.58 \pm 0.01$ & $1.42 \pm 0.01$ & $1.60 \pm 0.02$ \\
\hline OM (\%) & $2.35 \pm 0.07$ & $2.52 \pm 0.01$ & $2.24 \pm 0.02$ & $3.76 \pm 0.03$ & $12.34 \pm 0.02$ & $4.73 \pm 0.02$ & $8.58 \pm 0.04$ \\
\hline Total N (\%) & $0.04 \pm 0.02$ & $0.03 \pm 0.02$ & $0.05 \pm 0.01$ & $0.08 \pm 0.02$ & $0.12 \pm 0.02$ & $0.09 \pm 0.05$ & $0.07 \pm 0.02$ \\
\hline $\mathrm{C} / \mathrm{N}$ ratio & $30.25 \pm 0.13$ & $39.72 \pm 0.08$ & $16.65 \pm 0.11$ & $20.81 \pm 0.07$ & $21.48 \pm 0.12$ & $17.91 \pm 0.09$ & $22.93 \pm 0.12$ \\
\hline Available P (mg kg $\left.{ }^{-1}\right)$ & $52.01 \pm 0.08$ & $45.38 \pm 0.09$ & $24.45 \pm 0.22$ & $56.98 \pm 0.11$ & $30.64 \pm 0.19$ & $53.53 \pm 0.13$ & $20.35 \pm 0.22$ \\
\hline B. D $\left(\mathrm{g} \mathrm{cm}^{-3}\right)$ at $0-2 \mathrm{~cm}$ & $1.96 \pm 0.01$ & $1.68 \pm 0.02$ & $1.45 \pm 0.02$ & $1.54 \pm 0.01$ & $1.32 \pm 0.02$ & $1.49 \pm 0.03$ & $1.43 \pm 0.02$ \\
\hline $\mathrm{CO}_{3}^{2-}\left(\mathrm{mg} \mathrm{kg}^{-1}\right)$ & $2.40 \pm 0.01$ & $9.87 \pm 0.02$ & $10.1 \pm 0.05$ & $18.58 \pm 0.04$ & $14.24 \pm 0.02$ & $12.19 \pm 0.05$ & $16.8 \pm 0.02$ \\
\hline $\mathrm{NO}_{3}^{-}\left(\mathrm{mg} \mathrm{kg}^{-1}\right)$ & $6.80 \pm 0.05$ & $9.15 \pm 0.04$ & $7.8 \pm 0.02$ & $8.46 \pm 0.03$ & $12.67 \pm 0.09$ & $7.99 \pm 0.03$ & $10.36 \pm 0.03$ \\
\hline $\mathrm{NH}_{4}^{+}\left(\mathrm{mg} \mathrm{kg}^{-1}\right)$ & $0.01 \pm 0.03$ & $0.03 \pm 0.01$ & $0.08 \pm 0.02$ & $0.06 \pm 0.01$ & $0.10 \pm 0.01$ & $0.18 \pm 0.02$ & $0.23 \pm 0.01$ \\
\hline $\mathrm{SO}_{4}{ }^{2-}\left(\mathrm{mg} \mathrm{kg}^{-1}\right)$ & $24.00 \pm 0.11$ & $28.25 \pm 0.21$ & $47.87 \pm 0.19$ & $45.88 \pm 0.10$ & $61.12 \pm 0.11$ & $80.05 \pm 0.22$ & $44.14 \pm 0.21$ \\
\hline $\mathrm{PO}_{4}{ }^{3-}\left(\mathrm{mg} \mathrm{kg}^{-1}\right)$ & $13.40 \pm 0.06$ & $16.27 \pm 0.13$ & $18.80 \pm 0.06$ & $33.9 \pm 0.12$ & $20.07 \pm 0.08$ & $46.71 \pm 0.07$ & $14.52 \pm 0.07$ \\
\hline $\mathrm{Cl}^{-}\left(\mathrm{mg} \mathrm{kg}^{-1}\right)$ & $6.90 \pm 0.11$ & $8.00 \pm 0.02$ & $9.63 \pm 0.08$ & $37.00 \pm 0.14$ & $9.16 \pm 0.10$ & $14.31 \pm 0.12$ & $4.78 \pm 0.03$ \\
\hline Ex. Ca $\left(\mathrm{Cmolkg}^{-1}\right)$ & $5.56 \pm 0.04$ & $4.95 \pm 0.02$ & $6.65 \pm 0.06$ & $6.51 \pm 0.02$ & $8.15 \pm 0.02$ & $5.27 \pm 0.02$ & $13.21 \pm 0.07$ \\
\hline Ex. $\mathrm{Mg}\left(\mathrm{Cmolkg}^{-1}\right)$ & $2.80 \pm 0.16$ & $2.20 \pm 0.01$ & $2.49 \pm 0.02$ & $8.46 \pm 0.02$ & $13.78 \pm 0.09$ & $9.16 \pm 0.05$ & $13.87 \pm 0.12$ \\
\hline Ex. $\mathrm{Na}\left(\mathrm{Cmolkg}^{-1}\right)$ & $0.13 \pm 0.01$ & $0.12 \pm 0.02$ & $0.07 \pm 0.02$ & $0.09 \pm 0.01$ & $0.05 \pm 0.01$ & $0.13 \pm 0.02$ & $0.06 \pm 0.02$ \\
\hline Ex. K $\left(\mathrm{Cmolkg}^{-1}\right)$ & $0.02 \pm 0.01$ & $0.08 \pm 0.02$ & $0.06 \pm 0.02$ & $0.52 \pm 0.02$ & $0.28 \pm 0.02$ & $0.17 \pm 0.02$ & $0.14 \pm 0.02$ \\
\hline Ex. A $\left(\mathrm{Cmolkg}^{-1}\right)$ & $1.23 \pm 0.01$ & $1.16 \pm 0.02$ & $0.91 \pm 0.02$ & $0.91 \pm 0.02$ & $0.88 \pm 0.01$ & $1.25 \pm 0.02$ & $0.69 \pm 0.02$ \\
\hline ECEC $\left(\mathrm{Cmolkg}^{-1}\right)$ & $9.74 \pm 0.03$ & $8.50 \pm 0.06$ & $10.17 \pm 0.16$ & $16.48 \pm 0.04$ & $22.8 \pm 0.09$ & $15.98 \pm 0.02$ & $27.98 \pm 0.10$ \\
\hline
\end{tabular}

\subsection{Effects of Organic Fertilizers Application on Growth / Yield of Maize Plants}

The effects of the organic fertilizers application on maize growth and yield presented in Table 4 revealed the mean plant heights were $34.67 \pm 1.69$ and $26.61 \pm 1.60 \mathrm{~cm}$ (EC) compared to $113.77 \pm 90.39$ and $138.79 \pm 90.93 \mathrm{~cm}(\mathrm{EPM})$ and $102.73 \pm 92.28$ and $137.94 \pm 107.51 \mathrm{~cm}(\mathrm{ECM})$ during $1 \mathrm{st}$ and 2nd C. S respectively. The highest average number of leaves $(13.1 \pm 1.20)$ was observed in the plants grown on EPM followed by ECM plots (11.85 \pm 0.50$)$ while EC had the least $(6.5 \pm 1.15)$ average number of leaves (Table 4).

Table 4. Effect of organic fertilizer application on growth/yield of maize plants.

\begin{tabular}{|c|c|c|c|c|c|}
\hline Site & Av. Plant Ht. (cm) & Av. LA $\left(\mathrm{cm}^{2}\right)$ & Av. No. of Leaves & Av. Stem girth (cm) & Av. LAI \\
\hline \multicolumn{6}{|c|}{ 1st Cropping Season } \\
\hline $\mathrm{EC}$ & $34.67 \pm 1.69$ & $108.9 \pm 27.22$ & $7.2 \pm 7.51$ & $3.77 \pm 0.55$ & $6.12 \pm 0.45$ \\
\hline EPM & $113.77 \pm 90.39$ & $634.36 \pm 50.31$ & $12.28 \pm 0.95$ & $6.55 \pm 0.01$ & $51.73 \pm 5.26$ \\
\hline ECM & $102.73 \pm 92.28$ & $515.7 \pm 20.45$ & $10.83 \pm 2.01$ & $5.50 \pm 0.07$ & $38.92 \pm 3.20$ \\
\hline \multicolumn{6}{|c|}{ 2nd Cropping Season } \\
\hline $\mathrm{EC}$ & $26.61 \pm 1.60$ & $80.32 \pm 13.54$ & $6.5 \pm 1.15$ & $3.32 \pm 0.11$ & $3.46 \pm 0.11$ \\
\hline EPM & $138.79 \pm 90.93$ & $707.74 \pm 37.51$ & $13.1 \pm 1.20$ & $7.98 \pm 0.05$ & $60.45 \pm 8.00$ \\
\hline
\end{tabular}


Table 4. Continued.

\begin{tabular}{|c|c|c|c|c|c|c|}
\hline Site & $\begin{array}{l}\text { Fresh Corn } \\
\text { EarMean Wt (g) }\end{array}$ & HS. Wt (g) & Av. GNC--1 & $\begin{array}{l}\text { Grain Yield } \\
\text { (tonnes/acre) }\end{array}$ & $\begin{array}{l}\text { Stover Yield } \\
\text { (tonnes/acre) }\end{array}$ & H. I (GY/SY \pm GY) \\
\hline \multicolumn{7}{|c|}{ 1st Cropping Season } \\
\hline $\mathrm{EC}$ & $83.06 \pm 0.08$ & $19.00 \pm 0.10$ & $30.00 \pm 0.05(6)$ & $0.09 \pm 0.23$ & $0.15 \pm 0.60$ & $0.38 \pm 0.44$ \\
\hline EPM & $205.00 \pm 0.09$ & $32.17 \pm 0.05$ & $492.70 \pm 0.30(35)$ & $2.19 \pm 0.08$ & $1.50 \pm 0.01$ & $0.59 \pm 1.10$ \\
\hline ECM & $201.15 \pm 0.19$ & $33.00 \pm 0.10$ & $420.00 \pm 0.01(30)$ & $2.01 \pm 0.01$ & $1.93 \pm 0.01$ & $0.51 \pm 0.06$ \\
\hline \multicolumn{7}{|c|}{ 2nd Cropping Season } \\
\hline $\mathrm{EC}$ & $60.00 \pm 0.07$ & $16.13 \pm 0.10$ & $23.00 \pm 0.05$ (4) & $0.05 \pm 0.09$ & $0.21 \pm 0.25$ & $0.02 \pm 0.01$ \\
\hline $\mathrm{ECM}$ & $226.10 \pm 0.03$ & $38.00 \pm 0.06$ & $480.11 \pm 0.02(32)$ & $2.84 \pm 0.31$ & $1.79 \pm 1.71$ & $0.61 \pm 0.10$ \\
\hline
\end{tabular}

Key: Av.=average, Ht.=height, LA=leaf area, No.=number, LAI=leaf area index, Wt=weight, HS=hundred seed, GNC-1=Grain number per cob, HI=Harvest index, GY=Grain yield, SY=stover yield, Numbers in bracket=Numbers of row ear-1. Average of ten crops were used for each analysis except for seed weight and grain number per cob.

\section{Discussion}

\subsection{Microbial Counts of the Flood Plain During $1^{\text {st }} / 2^{\text {nd }}$ Cropping Seasons}

The total microbial counts of the soils are very important microbiological parameters and indicate the fertility and the activity of the soil. The increase in THBC populations during first 2 weeks could be explained to have co-incided with period of high rate decomposition stage when organic manure is transformed Jilani [14]. This result corroborates with the findings of Malik and Chauhan [15]. The values were statistically different at $\mathrm{P}=0.05$. The results of higher microbial abundance in the treated plots than in the control and higher in 2nd C. S than in the 1 st C. S. corroborates observations Mandic, Djukic, Beatovic, Zoran, Pesakovic and Stevovic [16]. Previous investigations have also demonstrated that animal compost increase microbial abundance by increasing the carbon pool of the soil thus improving the living conditions for indigenous microbial populations Zhen, Liu, Wang, Guo, Meng, Ding, $\mathrm{Wu}$, and Jiang [17].

\subsection{Effects of Organic Fertilizers on Growth and Yield of Maize}

The results of the greater ear mean weight (Ear $\mathrm{Wt}$ ) of plants on manured plots (ECM \& EPM) than on EC during both $\mathrm{C} . \mathrm{S}$ is consistent with the findings of Okoroafor, Okelola, Edeh, Emehute, Onu, Nwaneri, and Chinaka [18] who reported that poultry droppings gave higher mean weight of fresh cob of maize than the control. This research indicates that organic fertilizers improve the maize growth/yield and that the PM gave highest effect than the $\mathrm{CM}$ and control. The grain yields of maize obtained in this study, though lower than the standard real yield potential of 4.6 tha $^{-1}$ Pennington [19] had shown positive yield potential (more grain yield greater than stover yield) in both treatment plots. This signifies the importance of organic manuring of the flood plain in maize cultivation. Organic manuring could enhance special traits such as the ability to adapt to environmental stresses, disease existence which could have been enhanced by the rhizosphere microbes Nihorimbere, Ongena, Smargiassi, and Thonard [20].

\subsection{The Harvest Index}

The management of the plots (organic fertilizer application) had been suggested to contribute to the value of harvest index, $\mathrm{H}$. I by Pennington [19]. Thus the satisfactory values ( 0.51 to 0.63 ) of the studied plants especially on the EPM and ECM is explained and is within the recommended range $(0.50)$ as documented by Pennington [19]. Ion, Deu, Dumbrawa et al. [21] have recorded H. I similar (0.4) to these research findings.

\section{Conclusion}

A two year experiment at the Etinan flood plains provided a unique opportunity for the assessment of the effects of the utilization of organic fertilizers (cow dung and poultry droppings) on soil microbes, soil properties and maize growth/yield. The one time application of the organic fertilizers resulted in higher beneficial microbial density as well as increased physical and chemical properties (e.g. $\mathrm{TOC}$, soil organic matter, nutritive salts- $\mathrm{NO}_{3}, \mathrm{SO}_{4}, \mathrm{PO}_{4}$, $\mathrm{CO}_{3}$ in the 2 nd cropping season than the 1st. Thus, the utilization of composted organic fertilizers especially (poultry droppings) which showed (better effects) than cow dung on the wetland soil can indeed enhance sustainable agriculture.

\section{References}

[1] S. Devi, C. R. Sharma, and S. Singh, "on Microbial biodiversity in poultry and paddy straw wastes in composting systems", Brazilian Journal of Microbiology, 43 (1), pp 40-45, 2012.

[2] C. U. Etuk, "Influence of organic fertilizers on microbial activities and sustainable maize production in the tropical flood plain", Thesis submitted to the Postgraduate school, University of Uyo, Akwa Ibom, Nigeria, 2017.

[3] S. R. Imadi, K. Shazadi, A. Gul and K. R. Hakeem, Sustainable crop production system, In: K. Hakeem, M. Akhtar, (Edition), Plant Soil and Microbes, Springer: Cham Publishers, 2016, pp 103-116.

[4] P. K. Mohapatra, Environmental Microbiology, New Delhi: International Publishing House PVT Limited, 2008. 
[5] M. U. Imelda, V. E. Oshodeke and U. S. Akpan, "Determination of phosphorus fixing capacities and residual values of soils of Akwa Ibom State, Nigeria", Merit Research Journals, 2 (8), pp 96-103, 2014.

[6] I. C. Vinhal-Freitas, D. R. Wanger, A. S. Ferreira, G. F. Correa and B. Wendling, "Microbial and enzymatic activity in soil after organic composting", Revista Brasileira de Ciencia do Solo, 34, pp 757-764, 2010.

[7] J. Mercola, "How organic farming prevents the use of fertilizers",

Articles.mercola.com/sites/articles/archives/2013/07/...fertiliz er.aspx.Retrived on 2nd July 2016.

[8] R. C. Dubey, and D. K. Maheshwari, Practical Microbiology, New Delhi: Chand and Company Ltd., 2004, 352 pp.

[9] J. Traunfeld, "Soil amendments and fertilizers fertilizing guidelines included by plant groups", An Agricultural Extension Service Report submitted to the University of Maryland on April 2013, pp. 1-8.

[10] K. Cenciani, S. S. Freitas, S. A. M. Critter and C. Airoldi, "Enzymatic activity measured by microcabrimetry in soil amended with organic residues" Brazilian Science, Solo, 35, pp1167-1175, 2011.

[11] G. P. Robertson and P. M. Groffman, "Nitrogen transformation", In: Paul, E. A. (ed.), Soil Microbiology, Biochemistry and Ecology, New York: Springer, 2007, pp 341-364.

[12] O. M. Agbogidi and C. G. Okonmah, "Growth and yield of maize as influenced by organic manure type in a Niger Delta Environment", International Journal of Agriculture and Rural Development, 15 (1), pp 818- 824, 2012.

[13] R. R. Sokal, and F. J. Rohlf, Biometry: The Principal and Practice of Statistics in Biological Research, 2nd ed. USA: W. H. Freeman Company, 1981 pp 32.
[14] S. Jilani, "Municipal solid waste composting and its assessment for reuse in plant production", Pakistan Journal of Botany, 39 (1), pp 271-277, 2007.

[15] S. S. Malik and R. C. Chauhan, "Impact of organic farming in enhancing the soil microbial pool", In: Singh, M.; Singh, R. B. \& Hassan, M. I. (eds.). Climate Change and Biodiversity: Proceedings of Igu Rohtak Conference held on January 1, 2014.

[16] L. Mandic, D. Djukic, I. Beatovic, J. Zoran, M. Pesakovic and V. Stevovic, "Effect of different fertilizers on the microbial activity and productivity of soil under potato cultivation", African Journal of Biotechnology, 10 (36), pp 6954-6960, On online at http:www.academicjournals.org/AJB, 2011.

[17] Z. Zhen, H. Liu, N. Wang, L. Guo, J. Meng, N. Ding, G. Wu, and G. Jiang, "Effects of manure compost application on soil microbial community diversity and soil micronutrients in a temperate crop land in China", PLOS One, 9 (10), pp 108555, 2014.

[18] I. B. Okoroafor, O. E. Okelola, O. N. Edeh, V. C. Emehute, C. N. Onu, T. C. Nwaneri, and G. I. Chinaka, "Effect of organic manure on the growth and yield performance of maize in Ishiagwu, Ebonyi State, Nigeria", Journal of Agriculture and Veterinary Science, 5 (4), pp 28-31, 2013.

[19] D. Pennington, "Harvest index: A Predictor of corn stover yield", Available

at /msue.anr.msu.edu/news/harvest index a predictor of corn stover_yield/,2013), Retrieved on 2 nd Sept 2016.

[20] V. Nihorimbere, M. Ongena, M. Smargiassi, and P. Thonard, Biotechnology, Agronomy Society and Environment, 15 (2), pp 327-337, 2011.

[21] V. Ion, G. Dieu, M. Dumbrava, G. Temocico, I. N. Alecu, A. G Basa, and D. State, "Harvest index at maize in different growing condition", Romanian Biotechnological Letters, 20 (6), 10, 952-10, 960, 2015. 